\title{
ON THE EXISTENCE OF SMALL ANTICHAINS FOR DEFINABLE QUASI-ORDERS
}

\author{
RAPHAËL CARROY, BENJAMIN D. MILLER, \\ AND ZOLTÁN VIDNYÁNSZKY
}

\begin{abstract}
We generalize Kada's definable strengthening of Dilworth's characterization of the class of quasi-orders admitting an antichain of a given finite cardinality.
\end{abstract}

\section{INTRODUCTION}

A binary relation $R$ on a set $X$ is a quasi-order if it is reflexive and transitive. Two points $x, y \in X$ are $R$-comparable if $x R y$ or $y R x$, and $R$-incomparable otherwise. A set $Y \subseteq X$ is an $R$-chain if any two points of $Y$ are $R$-comparable, and an $R$-antichain if any two distinct points of $Y$ are $R$-incomparable.

Dilworth showed that if $k \in \mathbb{Z}^{+}, X$ is finite, and there is no $R$ antichain of cardinality $k+1$, then there is a cover $\left(C_{i}\right)_{i<k}$ of $X$ by $R$-chains (see [Dil50, Theorem 1.1]).

A subset of a topological space $X$ is Borel if it is in the $\sigma$-algebra generated by the topology $\tau_{X}$ of $X$, analytic if it is a continuous image of a closed subset of $\mathbb{N}^{\mathbb{N}}$, and $\aleph_{0}$-universally Baire if its preimage under any continuous function $\phi: 2^{\mathbb{N}} \rightarrow X$ has the Baire property.

Here we establish the following strengthening of Dilworth's theorem:

Theorem 1. Suppose that $k \in \mathbb{Z}^{+}, X$ is a Hausdorff space, and $R$ is an $\aleph_{0}$-universally-Baire quasi-order on $X$ whose incomparability relation is analytic. Then exactly one of the following holds:

(1) There is a cover $\left(C_{i}\right)_{i<k}$ of $X$ by Borel $R$-chains.

(2) There is an $R$-antichain of cardinality $k+1$.

The equivalence relation on $X$ associated with $R$ is that with respect to which two points $x, y \in X$ are equivalent if $x R y$ and $y R x$, and the strict relation associated with $R$ is that with respect to which two points $x, y \in X$ are related if $x R y$ but $\neg y R x$. Kada established the special case of Theorem 1 in which the strict quasi-order

2010 Mathematics Subject Classification. Primary 03E15, 28A05.

Key words and phrases. Antichain, chain, definable, dichotomy, quasi-order. 
is co-analytic and both the equivalence and incomparability relations are analytic (see [Kad89, Theorem $\left.1^{\prime}\right]$ ). Whereas his intricate argument relied heavily upon recursion-theoretic methods, we utilize only elementary ideas and the $\mathbb{G}_{0}$ dichotomy (see [KST99, Theorem 6.3]), which itself has a classical proof (see [Mil11, Theorem 8]).

A subset of an analytic Hausdorff space is $\boldsymbol{\Sigma}_{1}^{1}$ if it is analytic. More generally, for each $n \in \mathbb{Z}^{+}$, a subset of an analytic Hausdorff space is $\boldsymbol{\Pi}_{n}^{1}$ if its complement is $\boldsymbol{\Sigma}_{n}^{1}$, and $\boldsymbol{\Sigma}_{n+1}^{1}$ if it is a continuous image of a $\boldsymbol{\Pi}_{n}^{1}$ subset of an analytic Hausdorff space. A subset of an analytic Hausdorff space is $\boldsymbol{\Delta}_{n}^{1}$ if it is both $\boldsymbol{\Sigma}_{n}^{1}$ and $\boldsymbol{\Pi}_{n}^{1}$. Souslin's theorem ensures that the families of Borel and $\boldsymbol{\Delta}_{1}^{1}$ sets coincide (see, for example, [Kec95, Theorem 28.1]). The axiom of determinacy (AD) implies that the family of $\boldsymbol{\Delta}_{2 n+1}^{1}$ sets has a rich structural theory analogous to that of the Borel sets (see, for example, [Jac08]).

We also obtain the following analog of Theorem 1 under determinacy:

Theorem $2(\mathrm{AD})$. Suppose that $k \in \mathbb{Z}^{+}, n \in \mathbb{N}, X$ is an analytic Hausdorff space, and $R$ is a quasi-order on $X$ whose incomparability relation is $\Sigma_{2 n+1}^{1}$. Then exactly one of the following holds:

(1) There is a cover $\left(C_{i}\right)_{i<k}$ of $X$ by $\Delta_{2 n+1}^{1} R$-chains.

(2) There is an $R$-antichain of cardinality $k+1$.

In addition, we generalize Dilworth's theorem to arbitrary quasiorders on analytic Hausdorff spaces under the strengthening of determinacy where the players specify elements of $\mathbb{R}$ instead of $\mathbb{N}\left(\mathrm{AD}_{\mathbb{R}}\right)$ :

Theorem $3\left(\mathrm{AD}_{\mathbb{R}}\right)$. Suppose that $k \in \mathbb{Z}^{+}, X$ is an analytic Hausdorff space, and $R$ is a quasi-order on $X$. Then exactly one of the following holds:

(1) There is a cover $\left(C_{i}\right)_{i<k}$ of $X$ by $R$-chains.

(2) There is an $R$-antichain of cardinality $k+1$.

In $\S 1$, we establish Theorem 1. In $\S 2$, we describe the minor alterations to the proof necessary to obtain Theorems 2 and 3 , We work in the base theory ZF + DC throughout.

\section{The Classical CASE}

A binary relation $G$ on a set $X$ is a graph if it is irreflexive and symmetric. A $(Y$-) coloring of $G$ is a function $c: X \rightarrow Y$ such that $w G x \Longrightarrow c(w) \neq c(x)$ for all $w, x \in X$. The chromatic number of $G$, written $\chi(G)$, is the least cardinal $\kappa$ for which there is a $\kappa$-coloring of $G$ (if such a cardinal exists). We use $\chi_{\text {fin }}(G)$ to denote the supremum of the chromatic numbers of the graphs of the form $G \uparrow F$, where 
$F \subseteq X$ is a finite set. We use $G^{*}$ to denote the supergraph of $G$ with respect to which two points $x, y \in X$ are related if and only if there is a finite superset $F \subseteq X$ of $\{x, y\}$ such that $c(x) \neq c(y)$ for every $\chi_{\text {fin }}(G)$-coloring $c$ of $G \uparrow F$. Note that if $\chi_{\text {fin }}(G)=\aleph_{0}$, then $G=G^{*}$.

Proposition 4. Suppose that $X$ is a set, $G$ is a graph on $X$, and $G^{\prime} \subseteq$ $G^{*}$ is finite. Then there is a finite set $F \subseteq X$ containing $\bigcup_{i<2} \operatorname{proj}_{i}\left(G^{\prime}\right)$ such that every $\chi_{\text {fin }}(G)$-coloring $c$ of $G \uparrow F$ is a coloring of $\left(G^{\prime}\right)^{ \pm 1}$.

Proof. For all $(x, y) \in G^{\prime}$, fix a finite superset $F_{(x, y)} \subseteq X$ of $\{x, y\}$ such that $c(x) \neq c(y)$ for every $\chi_{\text {fin }}(G)$-coloring $c$ of $G \uparrow F_{(x, y)}$, and observe that the set $F=\bigcup_{(x, y) \in G^{\prime}} F_{(x, y)}$ is as desired.

A set $Y \subseteq X$ is a $G$-clique if any two distinct points of $Y$ are $G$ related, and $G$-independent if no two points of $Y$ are $G$-related.

Proposition 5. Suppose that $X$ is a set, $G$ is a graph on $X$, and $C \subseteq X$ is a finite $G^{*}$-clique. Then $|C| \leq \chi_{\text {fin }}(G)$.

Proof. By Proposition 4, there is a finite set $F \subseteq X$ containing $C$ such that $c \uparrow C$ is injective for every $\chi_{\mathrm{fin}}(G)$-coloring $c$ of $G \uparrow F$, in which case the pigeon-hole principle ensures that $|C| \leq \chi_{\mathrm{fin}}(G)$.

The horizontal sections of a set $R \subseteq X \times Y$ are the sets of the form $R^{y}=\{x \in X \mid x R y\}$, where $y \in Y$. The vertical sections are the sets of the form $R_{x}=\{y \in Y \mid x R y\}$, where $x \in X$.

Proposition 6. Suppose that $X$ is a set, $G$ is a graph on $X$ for which $\chi_{\text {fin }}(G)<\aleph_{0}, x, y \in X$, and there is a $G^{*}$-clique $C \subseteq G_{x}^{*} \cup G_{y}^{*}$ of cardinality $\chi_{\text {fin }}(G)$. Then $x G^{*} y$.

Proof. Proposition 4 yields a finite set $F \subseteq X$ containing $C \cup\{x, y\}$ such that $c \uparrow C$ is injective and $\forall w \in\{x, y\} \forall z \in C \cap G_{w}^{*} c(w) \neq c(z)$ for every $\chi_{\text {fin }}(G)$-coloring $c$ of $G \uparrow F$. But if $c$ is such a coloring, then $c(C)=\chi_{\mathrm{fin}}(G)$, so $c(x) \in c\left(C \cap G_{y}^{*}\right)$, thus $c(x) \neq c(y)$, hence $x G^{*} y$. $\otimes$

We use $\|_{R}, \equiv_{R}, \perp_{R}$, and $<_{R}$ to denote the comparability, equivalence, incomparability, and strict relations associated with $R$.

Proposition 7. Suppose that $X$ is a set and $R$ is a quasi-order on $X$. Then $R \backslash \perp_{R}^{*}$ is transitive.

Proof. Suppose, towards a contradiction, that there exist $x, y, z \in X$ for which $x\left(R \backslash \perp_{R}^{*}\right) y\left(R \backslash \perp_{R}^{*}\right) z$, as well as a finite set $F \subseteq X$ containing $\{x, z\}$ such that $c(x) \neq c(z)$ for every $\chi_{\text {fin }}\left(\perp_{R}\right)$-coloring $c$ of $\perp_{R} \uparrow F$. Then $x R z$, so $x$ and $z$ are not $\perp_{R}$-related, thus $\chi_{\text {fin }}\left(\perp_{R}\right)<\aleph_{0}$. For all $w \in\{x, z\}$, the fact that $w$ and $y$ are not $\perp_{R}^{*}$-related yields an $R$-chain 
$C_{w} \subseteq F \cup\{y\}$ containing $\{w, y\}$ for which $(F \cup\{y\}) \backslash C_{w}$ is a union of $\chi_{\text {fin }}\left(\perp_{R}\right)-1 R$-chains, and therefore does not contain an $R$-antichain of cardinality $\chi_{\mathrm{fin}}\left(\perp_{R}\right)$. Then the set $C=\left(C_{x} \cap R^{y}\right) \cup\left(C_{z} \cap R_{y}\right)$ is an $R$ chain containing $\{x, z\}$, so $(F \cup\{y\}) \backslash C$ is not a union of $\chi_{\mathrm{fin}}\left(\perp_{R}\right)-1 R$ chains, thus Dilworth's theorem yields an $R$-antichain $A \subseteq(F \cup\{y\}) \backslash C$ of cardinality $\chi_{\mathrm{fin}}\left(\perp_{R}\right)$. Fix $u \in A \cap C_{x}$ and $w \in A \cap C_{z}$. As $u, w \notin C$, it follows that neither $u R y$ nor $y R w$, so the fact that $C_{x}$ and $C_{z}$ are $R$-chains ensures that $w<_{R} y<_{R} u$, contradicting the fact that $A$ is an $R$-antichain.

Define $[x, y]_{R}=\{z \in X \mid x R z R y\}$ and $(x, y]_{R}=[x, y]_{R} \backslash[x]_{\equiv_{R}}$. We use $\frown$, $\sqsubseteq$, and $(i)$ to denote concatenation, extension, and the sequence of length one whose sole entry is $i$. Fix sequences $s_{n} \in 2^{n}$ that are dense in $2^{<\mathbb{N}}$, in the sense that $\forall s \in 2^{<\mathbb{N}} \exists n \in \mathbb{N} s \sqsubseteq s_{n}$, and define $\mathbb{G}_{0}=\left\{\left(s_{n} \frown(i) \frown c, s_{n} \frown(1-i) \frown c\right) \mid c \in 2^{\mathbb{N}}, i<2\right.$, and $\left.n \in \mathbb{N}\right\}$.

Proposition 8. Suppose that $X$ is a topological space, $R$ is an $\aleph_{0}$ universally-Baire quasi-order on $X$ that does not have antichains of arbitrarily large finite cardinality, and $\perp_{R}^{*}$ is $\aleph_{0}$-universally Baire. Then there is no continuous homomorphism $\phi: 2^{\mathbb{N}} \rightarrow X$ from $\mathbb{G}_{0}$ to $\perp_{R}^{*}$.

Proof. As Dilworth's theorem ensures that $\chi_{\mathrm{fin}}\left(\perp_{R}\right)<\aleph_{0}$, it is sufficient to show that if $\phi: 2^{\mathbb{N}} \rightarrow X$ is a continuous homomorphism from $\mathbb{G}_{0}$ to $\perp_{R}^{*}$, then there exists $x \in \phi\left(2^{\mathbb{N}}\right)$ for which there is a continuous homomorphism from $\mathbb{G}_{0}$ to $\perp_{R}^{*} \uparrow\left(\phi\left(2^{\mathbb{N}}\right) \cap\left(\perp_{R}^{*}\right)_{x}\right)$, since $\chi_{\text {fin }}\left(\perp_{R}\right)$ applications of this fact yield a $\perp_{R}^{*}$-clique of cardinality $\chi_{\text {fin }}\left(\perp_{R}\right)+1$, contradicting Proposition 5 .

Letting $G^{\prime}$ be the pullback of $\perp_{R}^{*}$ through $\phi \times \phi$, it is sufficient to find $c \in 2^{\mathbb{N}}$ for which $G_{c}^{\prime}$ has the Baire property and is not meager, as the proof of [KST99, Proposition 6.2] ensures that every $\mathbb{G}_{0}$-independent set with the Baire property is meager, so [KST99, Theorem 6.3] would then yield a continuous homomorphism $\psi: 2^{\mathbb{N}} \rightarrow G_{c}^{\prime}$ from $\mathbb{G}_{0}$ to $\mathbb{G}_{0} \uparrow G_{c}^{\prime}$ (although the existence of such a function also follows from a straightforward recursive construction), in which case the point $x=\phi(c)$ and the homomorphism $\phi \circ \psi$ are as desired.

Suppose, towards a contradiction, that every vertical section of $G^{\prime}$ with the Baire property is meager, and let $R^{\prime}$ be the pullback of $R$ through $\phi \times \phi$. As $\perp_{R}^{*}$ and $R$ are $\aleph_{0}$-universally Baire, the horizontal and vertical sections of $G^{\prime}$ and $R^{\prime}$ all have the Baire property. As $\perp_{R^{\prime}} \subseteq G^{\prime}$, every vertical section of $\perp_{R^{\prime}}$ is meager, and the KuratowskiUlam theorem (see, for example, Kec95, Theorem 8.41]) ensures that $\|_{R^{\prime}}$ is comeager, so $R^{\prime}$ is not meager.

Lemma 9. There exists $(b, d) \in \mathbb{G}_{0}$ for which $[b, d]_{R^{\prime}}$ is not meager. 
Proof. It is trivial to check that the binary relation $S^{\prime}$ on $2^{\mathbb{N}}$ given by $c S^{\prime} d \Longleftrightarrow \forall^{*} b \in 2^{\mathbb{N}}\left(b R^{\prime} c \Longrightarrow b R^{\prime} d\right)$ is a quasi-order, and for no $(d, c) \in \sim S^{\prime}$ is $(c, d]_{R^{\prime}}$ meager (see, for example, [Kec95, Proposition 8.26]). We can therefore assume that $\mathbb{G}_{0} \subseteq S^{\prime}$, so $\mathbb{G}_{0} \subseteq \equiv_{S^{\prime}}$. As the smallest equivalence relation on $2^{\mathbb{N}}$ containing $\mathbb{G}_{0}$ is $\mathbb{E}_{0}$ (by a straightforward inductive argument), it follows that $\mathbb{E}_{0} \subseteq \equiv_{S^{\prime}}$. As the Kuratowski-Ulam and Montgomery-Novikov theorems (see, for example, [Kec95, Theorem 16.1]) ensure that for all $s \in 2^{<\mathbb{N}}$, the corresponding set $B_{s}=\left\{c \in 2^{\mathbb{N}} \mid \forall^{*} b \in \mathcal{N}_{s} b R^{\prime} c\right\}$ has the Baire property, and $c \equiv_{S^{\prime}} d \Longleftrightarrow \forall s \in 2^{<\mathbb{N}}\left(c \in B_{s} \Longleftrightarrow d \in B_{s}\right)$ for all $c, d \in 2^{\mathbb{N}}$, the fact that every $\mathbb{E}_{0}$-invariant set with the Baire property is meager or comeager (see, for example, [Kec95, Theorem 8.47]) yields a comeager $\equiv_{S^{\prime}}$-class. Fixing $s, t \in 2^{<\mathbb{N}}$ with the property that $R^{\prime} \cap\left(\mathcal{N}_{s} \times \mathcal{N}_{t}\right)$ is comeager in $\mathcal{N}_{s} \times \mathcal{N}_{t}$, the Kuratowski-Ulam theorem implies that $\forall^{*} c \in \mathcal{N}_{t} \forall^{*} b \in \mathcal{N}_{s} b R^{\prime} c$, so $\forall^{*} b, c \in \mathcal{N}_{s} b R^{\prime} c$, thus there is an $\equiv_{R^{\prime}}$-class $C \subseteq 2^{\mathbb{N}}$ that is comeager in $\mathcal{N}_{s}$. But non-meager subsets of $2^{\mathbb{N}}$ with the Baire property are not $\mathbb{G}_{0}$-independent, and any pair $(b, d) \in \mathbb{G}_{0} \uparrow C$ is as desired.

As $b G^{\prime} d$, Proposition 7 ensures that $\forall c \in[b, d]_{R^{\prime}}\left(b G^{\prime} c\right.$ or $\left.c G^{\prime} d\right)$, so $G_{b}^{\prime}$ or $G_{d}^{\prime}$ is not meager, the desired contradiction.

Remark 10. A similar approach can be used to eliminate the need for multiple applications of the $\mathbb{G}_{0}$ dichotomy, and therefore the need to assume that $\operatorname{add}(\mathcal{M})<\kappa$, in [MV19] (see [Mil20, Propositions 1.6.17 and 1.6.19]).

Proposition 11. Suppose that $X$ is a set, $R$ is a quasi-order on $X$ that does not have antichains of arbitrarily large finite cardinality, $A \subseteq X$ is an $R$-antichain of cardinality $\chi_{\text {fin }}\left(\perp_{R}\right)$, and $Y \subseteq X$ is $\perp_{R}^{*}$-independent. Then there exists $x \in A$ for which $\{x\} \cup Y$ is $\perp_{R}^{*}$-independent.

Proof. Suppose, towards a contradiction, that there exists a function $\phi: A \rightarrow Y$ whose graph is contained in $\perp_{R}^{*}$. As Dilworth's theorem ensures that $\chi_{\text {fin }}\left(\perp_{R}\right)<\aleph_{0}$, it follows that $A$ is a maximal $R$-antichain, and is therefore the union of the sets $A^{\prime}=\left\{x \in A \mid A \cap R^{\phi(x)} \neq \emptyset\right\}$ and $A^{\prime \prime}=\left\{x \in A \mid A \cap R_{\phi(x)} \neq \emptyset\right\}$.

Lemma 12. The sets $A^{\prime}$ and $A^{\prime \prime}$ are disjoint.

Proof. Suppose, towards a contradiction, that there exists $x \in A^{\prime} \cap A^{\prime \prime}$, and fix $y, z \in A$ for which $y R \phi(x) R z$. As $A$ is an $R$-antichain, it follows that $y=z$, so $\phi(x) \equiv_{R} y$, thus the $\equiv_{R}$-invariance of $\perp_{R}^{*}$ yields that $\phi(x) \perp_{R}^{*} \phi(y)$, contradicting the $\perp_{R}^{*}$-independence of $Y$.

Lemma 13. If $w^{\prime}, x^{\prime} \in A^{\prime}$ and $\phi\left(x^{\prime}\right) R \phi\left(w^{\prime}\right)$, then $w^{\prime} \perp_{R}^{*} \phi\left(x^{\prime}\right)$. 
Proof. If $w^{\prime}$ and $\phi\left(x^{\prime}\right)$ are not $\perp_{R}^{*}$-related, then $w^{\prime} \|_{R} \phi\left(x^{\prime}\right)$, so Lemma 12 ensures that $w^{\prime}\left(R \backslash \perp_{R}^{*}\right) \phi\left(x^{\prime}\right)$. But the $\perp_{R^{-}}^{*}$ independence of $Y$ implies that $\phi\left(x^{\prime}\right)\left(R \backslash \perp_{R}^{*}\right) \phi\left(w^{\prime}\right)$, thus Proposition 7 yields that $w^{\prime}$ and $\phi\left(w^{\prime}\right)$ are not $\perp_{R}^{*}$-related, a contradiction.

Lemma 14. If $w^{\prime \prime}, x^{\prime \prime} \in A^{\prime \prime}$ and $\phi\left(w^{\prime \prime}\right) R \phi\left(x^{\prime \prime}\right)$, then $w^{\prime \prime} \perp_{R}^{*} \phi\left(x^{\prime \prime}\right)$.

Proof. If $w^{\prime \prime}$ and $\phi\left(x^{\prime \prime}\right)$ are not $\perp_{R}^{*}$-related, then $w^{\prime \prime} \|_{R} \phi\left(x^{\prime \prime}\right)$, so Lemma 12 ensures that $\phi\left(x^{\prime \prime}\right)\left(R \backslash \perp_{R}^{*}\right) w^{\prime \prime}$. But the $\perp_{R}^{*}$-independence of $Y$ implies that $\phi\left(w^{\prime \prime}\right)\left(R \backslash \perp_{R}^{*}\right) \phi\left(x^{\prime \prime}\right)$, thus Proposition 7 yields that $\phi\left(w^{\prime \prime}\right)$ and $w^{\prime \prime}$ are not $\perp_{R}^{*}$-related, a contradiction.

If $A^{\prime} \neq \emptyset$, then the fact that $Y$ is an $R$-chain yields $x^{\prime} \in A^{\prime}$ for which $\phi\left(x^{\prime}\right)$ is $\left(R \uparrow \phi\left(A^{\prime}\right)\right)$-minimal, so Lemma 13 ensures that $A^{\prime} \cup\left\{\phi\left(x^{\prime}\right)\right\}$ is an $\perp_{R}^{*}$-clique, and since Lemma 12 implies that $\phi\left(x^{\prime}\right) \notin A^{\prime}$, Proposition 5 yields that $\left|A^{\prime}\right|<\chi_{\mathrm{fin}}\left(\perp_{R}\right)$. Similarly, if $A^{\prime \prime} \neq \emptyset$, then the fact that $Y$ is an $R$-chain yields $x^{\prime \prime} \in A^{\prime \prime}$ for which $\phi\left(x^{\prime \prime}\right)$ is $\left(R \uparrow \phi\left(A^{\prime \prime}\right)\right)$-maximal, so Lemma 14 ensures that $A^{\prime \prime} \cup\left\{\phi\left(x^{\prime \prime}\right)\right\}$ is an $\perp_{R^{*}}^{*}$ clique, and since Lemma 12 implies that $\phi\left(x^{\prime \prime}\right) \notin A^{\prime \prime}$, Proposition 5 implies that $\left|A^{\prime \prime}\right|<\chi_{\mathrm{fin}}\left(\perp_{R}\right)$. It follows that $A^{\prime}$ and $A^{\prime \prime}$ are non-empty, so there are indeed $x^{\prime} \in A^{\prime}$ and $x^{\prime \prime} \in A^{\prime \prime}$ for which $\phi\left(x^{\prime}\right)$ is $\left(R \uparrow \phi\left(A^{\prime}\right)\right)$-minimal and $\phi\left(x^{\prime \prime}\right)$ is $\left(R \uparrow \phi\left(A^{\prime \prime}\right)\right)$-maximal. As $A \subseteq\left(\perp_{R}^{*}\right)_{\phi\left(x^{\prime}\right)} \cup\left(\perp_{R}^{*}\right)_{\phi\left(x^{\prime \prime}\right)}$ by Lemmas 13 and 14, Proposition 6 implies that $\phi\left(x^{\prime}\right) \perp_{R}^{*} \phi\left(x^{\prime \prime}\right)$, contradicting the $\perp_{R}^{*}$-independence of $Y$.

For each $k \in \mathbb{N}$, let $[X]^{k}$ denote the family of all subsets of $X$ of cardinality $k$, equipped with the topology generated by the sets of the form $\left\{F \in[X]^{k} \mid \exists \pi: F \hookrightarrow \mathcal{F} \forall x \in F x \in \pi(x)\right\}$, where $\mathcal{F} \in\left[\tau_{X}\right]^{k}$. Let $[X]^{\leq k}$ denote the disjoint union of the spaces of the form $[X]^{j}$, for $j \leq k$. Similarly, let $[X]^{<\aleph_{0}}$ denote the disjoint union of the spaces of the form $[X]^{k}$, for $k \in \mathbb{N}$. A set $Y \subseteq X$ punctures a family $\mathcal{F} \subseteq[X]^{<\aleph_{0}}$ if $F \cap Y \neq \emptyset$ for all $F \in \mathcal{F}$.

Proposition 15. Suppose that $X$ is a Hausdorff space, $G$ is an analytic graph on $X$ that admits a Borel coloring $c: X \rightarrow \mathbb{N}$, and $\mathcal{F} \subseteq[X]<\aleph_{0}$ $i s$ an analytic set with the property that for every $G$-independent set $Y \subseteq X$, the corresponding set $\{x \in X \mid\{x\} \cup Y$ is G-independent $\}$ punctures $\mathcal{F}$. Then every $G$-independent Borel subset of $X$ is contained in a $G$-independent Borel subset of $X$ that punctures $\mathcal{F}$.

Proof. For each natural number $k$ and $G$-independent set $Y \subseteq X$, we use $\mathcal{F}_{Y}^{k}$ to denote the family of sets $F \in \mathcal{F}$ with the property that $\mid\{x \in F \mid\{x\} \cup Y$ is not $G$-independent $\}|\geq| F \mid-k$. Note that $\mathcal{F}_{Y}^{0}=\emptyset$ and $\mathcal{F} \cap[X]^{\leq k} \subseteq \mathcal{F}_{Y}^{k}$. It is sufficient to show that for all $k \in \mathbb{N}$, every $G$-independent Borel set $B \subseteq X$ that punctures $\mathcal{F}_{B}^{k}$ is contained in 
a $G$-independent Borel set $C \subseteq X$ that punctures $\mathcal{F}_{C}^{k+1}$, as repeated application of this fact yields an increasing sequence of $G$-independent Borel supersets $B_{k} \subseteq X$ of any given $G$-independent Borel subset of $X$ that puncture $\mathcal{F}_{B_{k}}^{k}$, in which case the set $\bigcup_{k \in \mathbb{N}} B_{k}$ is as desired.

Suppose that $k \in \mathbb{N}$, we have already established the aforementioned fact strictly below $k$, and $B \subseteq X$ is a $G$-independent Borel set that punctures $\mathcal{F}_{B}^{k}$. Fix natural numbers $i_{j}$ such that $\forall i \in \mathbb{N} \exists^{\infty} j \in \mathbb{N} i=i_{j}$, and define $B_{0}^{\prime}=B$. Given $j \in \mathbb{N}$ and a $G$-independent Borel set $B_{j}^{\prime} \subseteq X$ that punctures $\mathcal{F}_{B_{j}^{\prime}}^{k}$, let $A_{j}^{\prime}$ be the set of $x \in X$ for which there exists $F \in \mathcal{F}$ disjoint from $B_{j}^{\prime}$ with the property that $x \in F$ and $\mid\left\{y \in F \backslash\{x\} \mid B_{j}^{\prime} \cup\{y\}\right.$ is not $G$-independent $\}|\geq| F \mid-(k+1)$. The fact that $B_{j}^{\prime}$ punctures $\mathcal{F}_{B_{j}^{\prime}}^{k}$ ensures that $B_{j}^{\prime} \cup\{x\}$ is $G$-independent for all $x \in A_{j}^{\prime}$, thus so too is $\left(A_{j}^{\prime} \cap c^{-1}\left(\left\{i_{j}\right\}\right)\right) \cup B_{j}^{\prime}$. As the latter set is analytic, it is contained in a $G$-independent Borel set (see, for example, the proof of [Mil11, Proposition 2]), in which case $k$ applications of the induction hypothesis yield a $G$-independent Borel set $B_{j+1}^{\prime} \subseteq X$ containing $\left(A_{j}^{\prime} \cap c^{-1}\left(\left\{i_{j}\right\}\right)\right) \cup B_{j}^{\prime}$ that punctures $\mathcal{F}_{B_{j+1}^{\prime}}^{k}$.

To see that the $G$-independent Borel set $C=\bigcup_{j \in \mathbb{N}} B_{j}^{\prime}$ punctures $\mathcal{F}_{C}^{k+1}$, observe that if $F \in \mathcal{F}_{C}^{k+1}$, then there exists $x \in F$ for which $C \cup\{x\}$ is $G$-independent, as well as $j \in \mathbb{N}$ for which $F \in \mathcal{F}_{B_{j}^{\prime}}^{k+1}$, and $j^{\prime} \geq j$ for which $i_{j^{\prime}}=c(x)$, in which case $B_{j^{\prime}}^{\prime} \cap F \neq \emptyset$ or $x \in B_{j^{\prime}+1}^{\prime}$. $\quad \square$

The Borel chromatic number of a graph $G$ on $X$ is the least cardinal $\chi_{\mathrm{B}}(G)$ of the form $|Y|$, where $Y$ is an analytic Hausdorff space for which there exists a Borel $Y$-coloring of $G$ (if such a space exists).

Proposition 16. Suppose that $X$ is a Hausdorff space and $R$ is a quasiorder on $X$ with the property that $\perp_{R}$ is analytic and $\chi_{B}\left(\perp_{R}^{*}\right) \leq \aleph_{0}$. Then $\chi_{B}\left(\perp_{R}^{*}\right)=\chi_{\text {fin }}\left(\perp_{R}\right)$.

Proof. As the case $\chi_{\mathrm{fin}}\left(\perp_{R}\right) \in\left\{1, \aleph_{0}\right\}$ is trivial, suppose that $k \in \mathbb{Z}^{+}$, we have already established the proposition for $\chi_{\text {fin }}\left(\perp_{R}\right) \leq k$, and $\chi_{\mathrm{fin}}\left(\perp_{R}\right)=k+1$. As $\perp_{R}^{*}$ is analytic, Propositions 11 and 15 yield an $\perp_{R}^{*}$-independent Borel set $B \subseteq X$ that intersects every $R$-antichain of cardinality $k+1$. As Dilworth's theorem ensures that $\chi_{\mathrm{fin}}\left(\perp_{R} \uparrow \sim B\right)=$ $k$, the induction hypothesis yields a Borel $k$-coloring $c$ of $\left(\perp_{R} \uparrow \sim B\right)^{*}$. Observe that $\perp_{R}^{*} \uparrow \sim B \subseteq\left(\perp_{R} \uparrow \sim B\right)^{*}$, for if $x, y \in \sim B$ and $F \subseteq X$ is a finite set containing $\{x, y\}$ such that $d(x) \neq d(y)$ for every $(k+1)$ coloring $d$ of $\perp_{R} \uparrow F$, then $F \backslash B$ is a finite set containing $\{x, y\}$ such that $d(x) \neq d(y)$ for every $k$-coloring $d$ of $\perp_{R} \uparrow(F \backslash B)$. In particular, it follows that the extension of $c$ to $X$ with constant value $k$ on $B$ is a Borel $(k+1)$-coloring of $\perp_{R}^{*}$. 
As every analytic subset of a topological space is $\aleph_{0}$-universally Baire (see, for example, [Kec95, Theorem 21.6]), Theorem 1 follows from Proposition 8, the $\mathbb{G}_{0}$ dichotomy, and Proposition 16.

\section{Generalizations under Determinacy}

Given an ordinal $\alpha$, a subset of a topological space $X$ is $\alpha$-Borel if it is in the closure of $\tau_{X}$ under complements and unions of length strictly less than $\alpha$. Given an aleph $\kappa$, a topological space is $\kappa$-Souslin if it is a continuous image of a closed subset of $\kappa^{\mathbb{N}}$.

For all $n>0$, let $\boldsymbol{\delta}_{n}^{1}$ denote the supremum of the lengths of wellorders of the form $R / \equiv_{R}$, where $R$ is a $\Delta_{n}^{1}$ quasi-order on an analytic Hausdorff space. The axiom of determinacy ensures that the $\boldsymbol{\Delta}_{2 n+1}^{1}$ and $\boldsymbol{\delta}_{2 n+1}^{1}$-Borel subsets of analytic Hausdorff spaces coincide. It also yields an aleph $\boldsymbol{\lambda}_{2 n+1}^{1}$ for which $\boldsymbol{\delta}_{2 n+1}^{1}=\left(\boldsymbol{\lambda}_{2 n+1}^{1}\right)^{+}$, and implies that the $\boldsymbol{\Sigma}_{2 n+1}^{1}$ and $\boldsymbol{\lambda}_{2 n+1}^{1}$-Souslin subsets of analytic Hausdorff spaces coincide (see, for example, Jac08).

A tree on a set $I$ is a set $T \subseteq I^{<\mathbb{N}}$ that is closed under initial segments, in the sense that $\forall t \in T \forall n<|t| t \uparrow n \in T$. A subtree of $T$ is a tree $S \subseteq T$ on $I$. A branch through $T$ is a sequence $x \in I^{\mathbb{N}}$ such that $\forall n \in \mathbb{N} x\lceil n \in T$. A tree is well-founded if it has no branches.

The pruning derivative associates with each tree $T$ on a set $I$ the subtree $T^{\prime}=\{t \in T \mid \exists i \in I t \frown(i) \in T\}$. The iterates of the pruning derivative are given by $T^{(0)}=T, T^{(\alpha+1)}=\left(T^{(\alpha)}\right)^{\prime}$ for all ordinals $\alpha$, and $T^{(\lambda)}=\bigcap_{\alpha<\lambda} T^{(\alpha)}$ for all limit ordinals $\lambda$. The pruning rank of $T$ is the least ordinal $\rho(T)$ for which $T^{(\rho(T))}=T^{(\rho(T)+1)}$. A straightforward induction shows that $T$ is well-founded if and only if $T^{(\rho(T))}=\emptyset$. For each $t \in T$, let $\rho_{T}(t)$ denote the largest ordinal for which $t \in T^{\left(\rho_{T}(t)\right)}$ (if such an ordinal exists).

An $(\alpha+1)$-Borel code for a subset of $X$ is a pair $(f, T)$, where $T$ is a well-founded tree on $\alpha \times \alpha$ and $f$ is a function associating to each sequence $t \in \sim T$ a subset of $X$ that is closed or open. Given such a code, we recursively define $f^{(\beta)}$ on $\sim T^{(\beta)}$ by setting $f^{(0)}=f$, letting $f^{(\beta+1)}$ be the extension of $f^{(\beta)}$ given by $f^{(\beta+1)}(t)=\bigcup_{\gamma<\alpha} \bigcap_{\delta<\alpha} f^{(\beta)}(t \frown((\gamma, \delta)))$ whenever $\rho_{T}(t)=\beta$ for all ordinals $\beta$, and defining $f^{(\lambda)}=\bigcup_{\beta<\lambda} f^{(\beta)}$ for all limit ordinals $\lambda$. The $(\alpha+1)$-Borel set coded by $(f, T)$ is $f^{(\rho(T))}(\emptyset)$.

The proof of Souslin's theorem shows that there is a function sending each pair of functions witnessing that a set and its complement are $\kappa$ Souslin to a $(\kappa+1)$-Borel code for the set. Under $\mathrm{AD}$, the coding lemma (see [Mos09, Lemma 7D.5]) and projective uniformization (see, for example, [Kec95, Theorem 39.9]) can be used to obtain a function 
sending each $\left(\boldsymbol{\lambda}_{2 n+1}^{1}+1\right)$-Borel code for a subset of an analytic Hausdorff space to a function witnessing that the encoded set is $\boldsymbol{\lambda}_{2 n+1}^{1}$-Souslin.

Proposition $17(\mathrm{AD})$. Suppose that $n \in \mathbb{N}, X$ is an analytic Hausdorff space, $G$ is a $\Sigma_{2 n+1}^{1}$ graph on $X$ that admits a $\boldsymbol{\Delta}_{2 n+1}^{1}$ coloring $c: X \rightarrow \lambda_{2 n+1}^{1}$, and $\mathcal{F} \subseteq[X]^{<\aleph_{0}}$ is a $\Sigma_{2 n+1}^{1}$ set with the property that for every $G$-independent set $Y \subseteq X$, the corresponding set $\{x \in X \mid$ $\{x\} \cup Y$ is $G$-independent $\}$ punctures $\mathcal{F}$. Then every $G$-independent $\Delta_{2 n+1}^{1}$ subset of $X$ is contained in a $G$-independent $\Delta_{2 n+1}^{1}$ subset of $X$ that punctures $\mathcal{F}$.

Proof. We proceed essentially as in the proof of Proposition 15. The first paragraph remains unchanged. The induction beginning in the second paragraph, however, has length $\lambda_{2 n+1}^{1}$ instead of $\omega$, which is problematic because naively applying [Mil11, Proposition 2] at each stage of the induction requires too large a fragment of the axiom of choice. This problem can be alleviated by using the above remarks to keep track of codes for the sets $B_{j}^{\prime}$ that are built along the way, which can be achieved because the proof of [Mil11, Proposition 2] utilizes little more than Souslin's theorem.

Proposition 17 gives rise to an analogous version of Proposition 16. As every subset of a topological space is $\aleph_{0}$-universally Baire under AD (see, for example, Mos09, Theorem 7D.2]), this can be combined with Proposition 8 and Kanovei's generalization of the $\mathbb{G}_{0}$ dichotomy (see [Kan97], although the elementary proof of [Mil11, Theorem 8] can be adapted to obtain the special cases we need by keeping track of codes as above) to establish Theorem 2.

By eliminating the outer induction and the use of [Mil11, Proposition $2]$ in the proof of Proposition [15, one obtains a proof of the weaker result without definability conditions on the sets involved. Moreover, this proof trivially generalizes to colorings $c: X \rightarrow \kappa$, for any aleph $\kappa$, and gives rise to an analogous version of Proposition 16. As a result of Woodin's ensures that every subset of an analytic Hausdorff space is $\kappa$-Souslin, for some aleph $\kappa$, under $\mathrm{AD}_{\mathbb{R}}$ (see, for example, Kan03, Theorem 32.23]), this can be combined with Proposition 8 and the weakening of Kanovei's generalization of the $\mathbb{G}_{0}$ dichotomy in which there are no definability constraints on the coloring (which follows from the simplification of the proof of [Mil11, Theorem 8] in which the use of Souslin's theorem is eliminated) to establish Theorem 3 . 


\section{REFERENCES}

[Dil50] R.P. Dilworth, A decomposition theorem for partially ordered sets, Ann. of Math. (2) 51 (1950), 161-166. MR 32578

[Jac08] S.C. Jackson, Suslin cardinals, partition properties, homogeneity. Introduction to Part II, Games, scales, and Suslin cardinals. The Cabal Seminar. Vol. I, Lecture Notes in Logic, vol. 31, Association for Symbolic Logic, Chicago, IL, 2008, pp. 273-313. MR 2463617

[Kad89] K. Kada, Une version borélienne du théorème de Dilworth, Ph.D. thesis, University of Paris 6, 1989.

[Kan97] V. Kanovei, Two dichotomy theorems on colourability of non-analytic graphs, Fund. Math. 154 (1997), no. 2, 183-201, European Summer Meeting of the Association for Symbolic Logic (Haifa, 1995). MR 1477757 (98m:03103)

[Kan03] A. Kanamori, The higher infinite, second ed., Springer Monographs in Mathematics, Springer-Verlag, Berlin, 2003, Large cardinals in set theory from their beginnings. MR 1994835

[Kec95] A.S. Kechris, Classical descriptive set theory, Graduate Texts in Mathematics, vol. 156, Springer-Verlag, New York, 1995. MR 1321597 (96e:03057)

[KST99] A.S. Kechris, S.J. Solecki, and S. Todorcevic, Borel chromatic numbers, Advances in Mathematics 141 (1999), no. 1, 1-44. MR 1667145

[Mil11] B.D. Miller, Dichotomy theorems for countably infinite dimensional analytic hypergraphs, Ann. Pure Appl. Logic 162 (2011), no. 7, 561-565. MR 2781095

[Mil20] - Structural dichotomy theorems in descriptive set theory, available at http://www.logic.univie.ac.at/ millerb45/dichotomies.pdf 2020.

[Mos09] Y.N. Moschovakis, Descriptive set theory, second ed., Mathematical Surveys and Monographs, vol. 155, American Mathematical Society, Providence, RI, 2009. MR 2526093

[MV19] B.D. Miller and Z. Vidnyánszky, On the existence of large antichains for definable quasi-orders, The Journal of Symbolic Logic (2019), 1-8.

Raphaël Carroy, Dipartimento di Matematica "Giuseppe Peano", Università di Torino, Palazzo Campana, Via Carlo Alberto 10, 10123 TORINO, ITALIA

E-mail address: raphael.carroy@unito.it

URL: http://www.logique.jussieu.fr/ carroy/indexeng.html

Benjamin D. Miller, Kurt Gödel Research Center for MathematiCal Logic, Universität Wien, Augasse 2-6, 1090 Wien, Austria

E-mail address: benjamin.miller@univie.ac.at

$U R L:$ http://www.logic.univie.ac.at/benjamin.miller

Zoltán Vidnyánszky, Kurt Gödel Research Center for MathematiCal Logic, Universität Wien, Augasse 2-6, 1090 Wien, Austria

E-mail address: zoltan.vidnyanszky@univie.ac.at

URL: http://www.logic.univie.ac.at/ vidnyanszz77 